UCRL-JC-125939

Preprint

\title{
A Study of Anisotropy of Spin Cast and Vapor Deposited Polyimide Films Using Internal Reflection Techniques
}

\author{
V. Liberman \\ V. Malba \\ A. F. Bernhardt \\ This paper was prepared for submittal to \\ Materials Research Society Fall Meeting \\ Boston, MA \\ December 2-6, 1996
}

November 1996

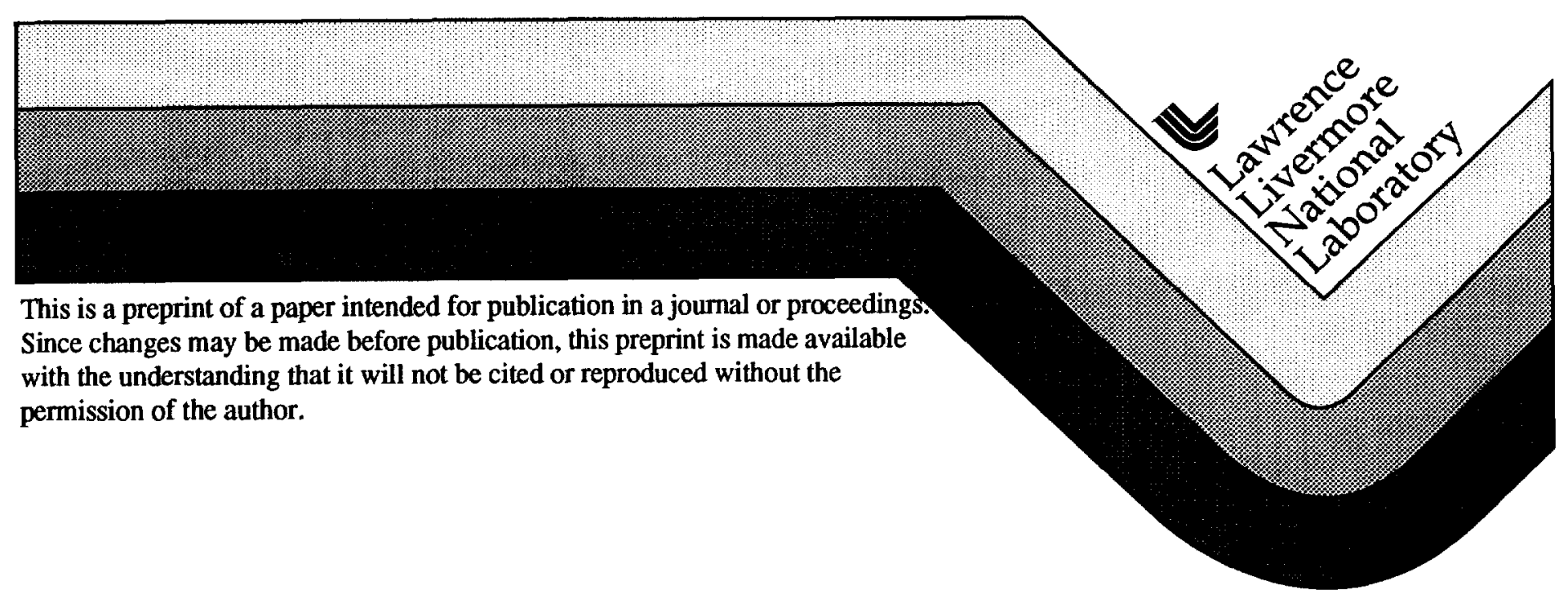




\section{DISCLAIMER}

This document was prepared as an account of work sponsored by an agency of the United States Government. Neither the United States Government nor the University of California nor any of their employees, makes any warranty, express or implied, or assumes any legal liability or responsibility for the accuracy, completeness, or usefulness of any information, apparatus, product, or process

disclosed, or represents that its use would not infringe privately owned rights. Reference herein to any specific commercial product, process, or service by trade name, trademark, manufacturer, or otherwise, does not necessarily constitute or imply its endorsement, recommendation, or favoring by the United States Government or the University of California. The views and opinions of authors expressed herein do not necessarily state or reflect those of the United States Government or the University of California, and shall not be used for advertising or product endorsement purposes. 


\title{
A STUDY OF ANISOTROPY OF SPIN CAST AND VAPOR DEPOSITED POLYIMIDE FILMS USING INTERNAL REFLECTION TECHNIQUES
}

\author{
VLADIMIR LIBERMAN, VINCENT MALBA AND ANTHONY F. BERNHARDT
}

Lawrence Livermore National Lab, P. O. Box 808, L-271, Livermore, CA 94550

\begin{abstract}
We have compared anisotropy of spin cast and vapor deposited polyimide (VDP) films, using internal reflection infrared spectroscopy. The films were deposited directly on the internal reflection element. We find that spin cast films are more anisotropic than their VDP counterparts, with the polyimide chains tending to align parallel to the substrate. Both films are found to contain more and less ordered regions. Within the ordered regions, the plane of the phenyl ring tends to align parallel to the substrate.
\end{abstract}

\section{INTRODUCTION}

Because of its excellent thermal, mechanical and electrical properties, polyimide has received wide use in microelectronics as a dielectric material. Commercial polyimides for microelectronics applications are provided as solutions of a polyamic acid, that has been prepared by condensation of a dianhydride and a diamine. A common aromatic polyamic acid is formed by condensation of pyromellitic dianhydride (PMDA) and oxydianiline (ODA) in n-methyl pyrrolidone (NMP) solution. The polyamic acid solution is typically spin cast onto an appropriate substrate. Then, a thermal curing process is used to drive off the solvent and initiate imide ring closure which produces the insoluble polyimide. The resultant spin coated films are inherently planarizing and are thus not well suited for dielectric applications which require conformal sidewall coverages.

An alternative polyimide deposition method based on vapor deposition polymerization (VDP) allows formation of solventless films through vacuum coevaporation of a dianhydride and a diamine. 1,2 The resultant films are subsequently cured to form a polyimide coating. In a previous work, we have demonstrated a robust VDP polyimide process and its integration into a multichip module packaging process. Cured VDP films were shown to exhibit thermal stability, chemical resistance and dielectric breakdown properties comparable to their spin cast counterparts.

Although electrical, mechanical and thermal properties of spin cast PMDA-ODA polyimide films have been studied extensively ${ }^{3}$, these properties are usually measured only in a single plane. For instance, dielectric constant of thin films is typically obtained through parallel-plate capacitor measurements with the electric field normal to the film plane. Thermal expansion measurements have been traditionally performed in the direction along the film plane.

Recent work has shown that both electrical ${ }^{4}$ and thermal expansion ${ }^{5}$ properties of spin cast polyimides display significant anisotropy. The amount of anisotropy is partially determined by the rigidity of the polymer chains with the rigid poly ( $p$ phenylene biphenyltetracarboximide) (BPDA-PPD) type polyimides displaying more anisotropy than the semi--flexible PMDA-ODA type polyimides. This anisotropy is believed to arise from the tendency of the spin cast films, to align preferentially along the surface of the substrates during spin casting and subsequent imidization 6 .

Due to the nature of the deposition process, one would expect that vapor deposited polyimides display less anisotropy than their spin cast counterparts. Indeed, 
previous work, based on refractive index anisotropy measurements at $632.8 \mathrm{~nm}^{2}$, suggested that vapor deposited PMDA-ODA films are more isotropic. In a previous work ${ }^{7}$, we have performed extensive characterization of the refractive index anisotropy of our VDP films as compared to spin cast films. For the range of film thicknesses from $0.6 \mu \mathrm{m}$ to $5 \mu \mathrm{m}$, the following conclusions were drawn:

1) no in-plane anisotropy of the refractive index could be detected, i.e., $n_{x}=n_{y}$;

2) no thickness dependence is observed for either in- or out-of plane refractive index;

3) For a typical $2 \mu \mathrm{m}$ cured VDP film, $n_{x y}$ is $1.722, n_{z}$ is 1.662 , and index anisotropy, $\Delta=n_{z}-n_{x y}$, is 0.060 . By comparison, a $2 \mu \mathrm{m}$ cured spin coated 2545 film has $n_{x y}=$ $1.725, \mathrm{n}_{\mathrm{z}}=1.647$, and $\Delta=0.078$.

While this potentially reduced anisotropy of VDP films could have profound implications for their use in microelectronics, there has not, to our knowledge, been a study comparing molecular orientation differences in vapor deposited films and their spin cast counterparts.

Our work utilized Attenuated Total Reflectance Fourier Transform Infrared (ATR FTIR) spectroscopy to compare differences in molecular chain orientation of our VDP PMDA-ODA films with their spin cast counterparts. As described below, ATR FTIR spectroscopy is ideally suited to the study molecular orientation since both in-plane and out-of plane molecular vibrations can be easily detected. Furthermore, by depositing both spin cast and VDP films directly on the ATR internal reflection element (IRE), highly accurate measurements can be obtained, allowing the detection of even small differences in molecular orientation.

\section{EXPERIMENTAL}

\section{Vapor Deposition of Polyimides}

Since the VDP deposition system has been described in detail elsewhere, we give here only a brief summary 7 . The deposition system is a modified bell-jar vacuum evaporator with a three-sample rotating planetary holder. The two monomers, pyromellitic dianhydride (PMDA) and oxydianiline (ODA), are evaporated from source ovens, individually heated by quartz lamps and controlled by a feedback loop from quartz thickness monitors. With chamber pressure not exceeding $1 \times 10^{-5}$ Torr for the highest deposition rates, the evaporation is a line-of-sight process. During deposition the temperature of the sample holders rises to $\leq 50^{\circ} \mathrm{C}$ due to radiative heating from the deposition sources. At the completion of a deposition run, the samples are removed from the chamber and cured ex-situ in an inert gas atmosphere. The curing schedule for the VDP films is as follows: soft bake at $150^{\circ} \mathrm{C}$, a slow ramp up to $260^{\circ}$ $\mathrm{C}$, a one hour final cure, followed by a cool down. For comparison, the spin cast films are cured with the following schedule: 10 minute pre-bake at $90^{\circ} \mathrm{C}, 30$ minute bake at $120^{\circ} \mathrm{C}, 5^{\circ} \mathrm{C} / \mathrm{min}$ ramp to $400^{\circ} \mathrm{C}, 30$ minute high temperature bake and a slow cool down. For spin cast uncured polyamic acid analysis, the films are baked for $1 \mathrm{hr}$ at $95 \mathrm{C}$ to remove most of the solvent trapped within the film.

FTIR measurements were performed using Nicolet 510 spectrometer with a variable angle Spectra-Tech ATR accessory and a ZnSe wire grid polarizer. For each spectrum, 300 scans were acquired at $4 \mathrm{~cm}^{-1}$ resolution. Prior to depositing a film onto the IRE, background spectra were collected at different polarizations with the uncoated IRE. All the ATR FTIR spectra presented here have been corrected for the number of reflections. 


\section{ATR-FTIR}

The basic ATR-FTIR setup, shown in Fig. 1, involves launching an infrared beam into an internal reflection element (IRE). The sample to be studied is placed in contact with the IRE. The angle of incidence at the IRE/sample interface should be chosen to exceed the critical angle ( $>45^{\circ}$ assuming $\mathrm{ZnSe}$ IRE and $n=1.7$ for polyimide). At each reflection point, an evanescent field is setup, which decays exponentially in the zdirection (away from the IRE/sample interface). Interaction of the evanescent field with the sample results in absorption bands. Great enhancement of the signal to noise ratio results and because of multiple IRE/sample reflections. If we separate the incident electric field into s- and p-components, $E_{s}$ and $E_{p}$, we may write in the coordinate system of Fig. 1:

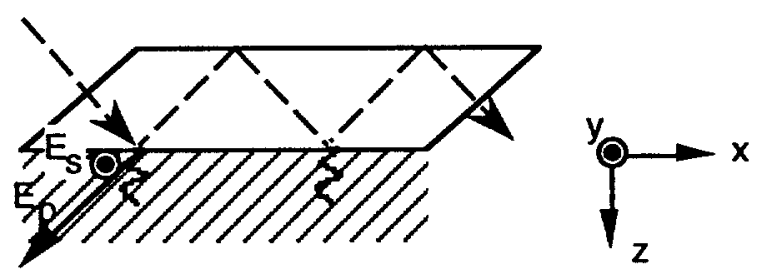

Fig. 1. Geometrical layout of the ATR FTIR setup, with the relevant electric fields.

$$
\left|E_{s}\right|=\left|E_{y}\right| \quad \text { and }\left|E_{p}\right|=\left(\left|E_{z}\right|^{2}+\left|E_{x}\right|^{2}\right)^{1 / 2}
$$

The ability to detect molecular vibrations whose transition moments are out of the plane of the film $\left(E_{z}\right)$ can be defined by the contrast ratio $E_{z} / E_{x}$. For the evanescent field at the interface on the polyimide side,

$$
\left|\frac{E_{z}}{E_{x}}\right|=\frac{\sin \theta}{\sqrt{\sin ^{2} \theta-\left(n_{\mathrm{PI}} / n_{\mathrm{IRE}}\right)^{2}}}
$$

where $n_{P I}$ is the refractive index of polyimide and $n_{I R E}$ is the refractive index of the IRE. For a ZnSe IRE and an incident angle of $60^{\circ},\left|\frac{E_{z}}{E_{x}}\right| \frac{a}{1.75 . ~ B y ~ c o m p a r i s o n, ~ f o r ~ a ~}$ transmission FTIR measurement, $\left|\frac{E_{z}}{E_{x}}\right|$ is 0.59 for a $60^{\circ}$ incident angle and 0.72 for an $85^{\circ}$ incident angle. Therefore, p-polarized absorption in the ATR FTIR signal is dominated by the out of plane electric field component, unlike a transmission FTIR measurement.

While ATR FTIR polyimide spectra can be obtained by depositing the film on a wafer and pressing the wafer against the crystal surface, the method has a number of drawbacks. First, because of the rigidity of the substrate, good optical contact cannot be obtained in more than one or two points. Furthermore, for a high refractive index substrate, such as silicon $(n=3.4)$ light can couple out from the IRE waveguide to the substrate in what is known as Frustrated Total Reflection (FTR). FTR can be especially pronounced at wavenumbers $<1000 \mathrm{~cm}^{-1}$, where the penetration depth of the evanescent wave can exceed the film thickness. This results in marked drop of the detected signal at the lower wavenumbers.

The above difficulties can be avoided if the films are deposited directly on the side of an IRE. While VDP films can be easily deposited on irregular masked surfaces, 
a special fixture needed to be designed for obtaining uniform spin coated films on the IRE surface (Fig. 2). The IRE is clamped in the fixture with nylon-tipped screws. A template covering the top of the IRE masks exit and entrance faces and serves as a dummy edge for spin casting the films. Using this fixture, uniform spin cast films could be deposited in the thickness range of 0.2 to $2 \mu \mathrm{m}$ with spin speeds up to $5000 \mathrm{rpm}$. For the work described here, $50 \times 10 \times 3 \mathrm{~mm} Z \mathrm{ZnSe}$ parallelogram IRE with a face angle of $60^{\circ}$ was used. At an incident angle of $59^{\circ}$, this geometry yielded 5 reflections per side. Because of intense IR absorption of polyimide, films with thicknesses $\leq 0.5 \mu \mathrm{m}$ could be analyzed with this geometry.

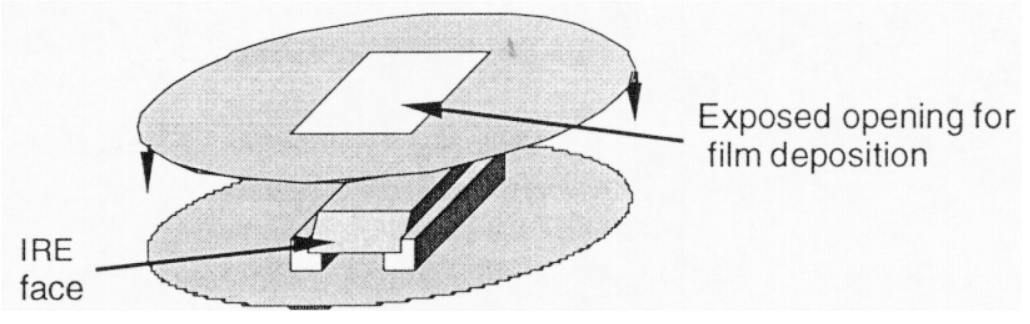

Fig. 2. Fixture used for depositing polyimide films directly onto an IRE

\section{RESULTS}

ATR spectra for a $0.5 \mu \mathrm{m}$ spin cast PI film are shown in Fig. 3. Similar spectra were obtained for a $0.41 \mu \mathrm{m}$ VDP film. The VDP film was deposited at a rate of $4 \mu \mathrm{m} / \mathrm{hr}$.

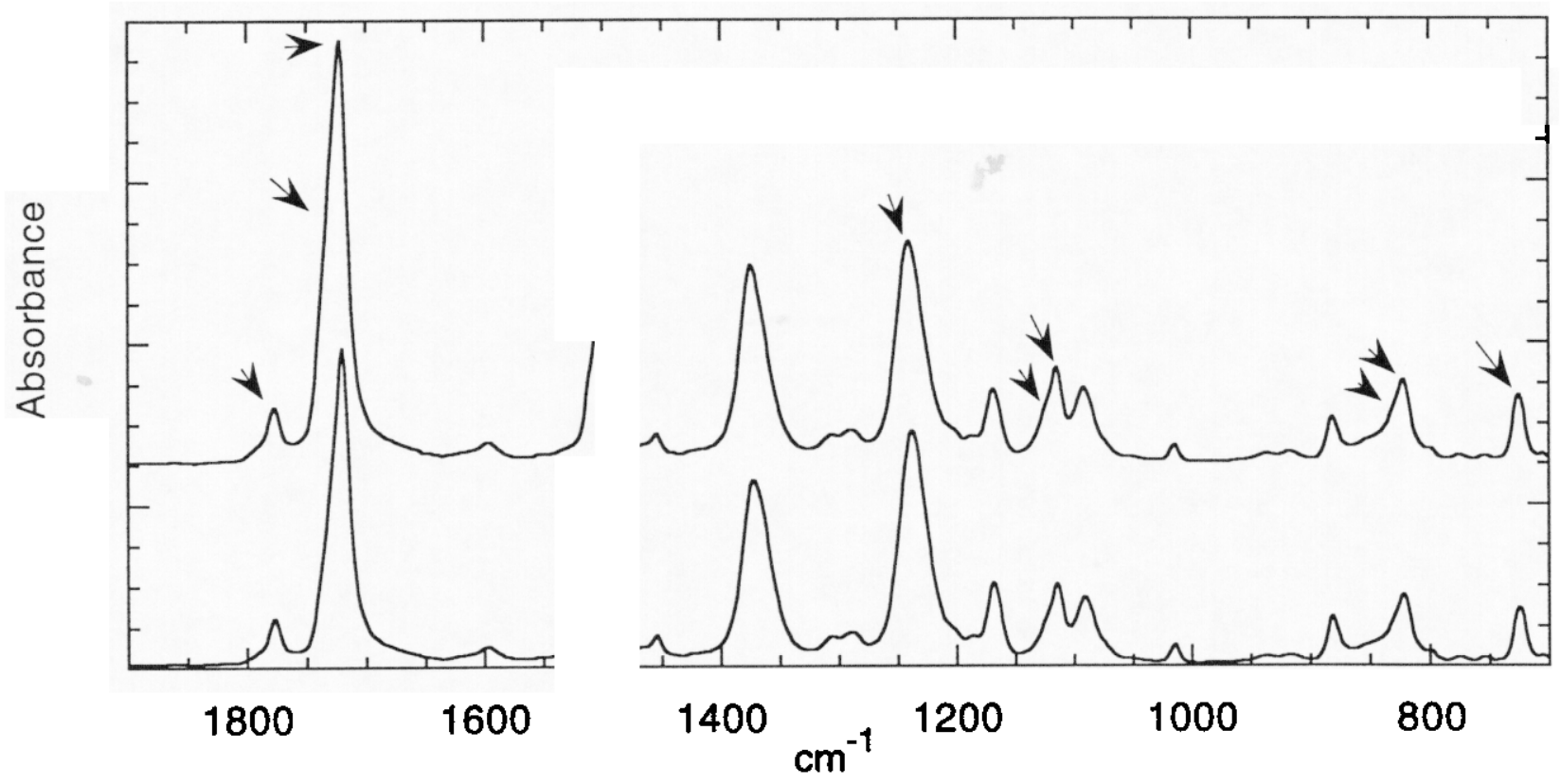

Fig. 3. ATR FTIR spectra of $0.5 \mu \mathrm{m}$ spin cast film deposited onto an IRE. Top: ppolarization, bottom: s-polarization. Arrows indicate positions of vibrational modes used for structural analysis (see text).

The anisotropy of the films was analyzed by following polyimide absorption peaks whose moment directions relative to the polymer chain have been assigned 
previously. ${ }^{7}$ After close examination of the FTIR spectra, such as in Fig. 3, several relevant peaks were found to consist of doublets. These overlapping peaks were deconvoluted using peak fitting software (PeakSolve, Galactic industries). The doublet behavior is believed to arise from the presence of ordered (0) regions and disordered (d) regions within the sample 8,9 . Table I shows the position of the peaks, marked in Fig. 3, with the doublet peaks labeled "o" or "d" for ordered and disordered regions, respectively. The second column of the table gives mode description and mode orientation relative to the polymer chain, according to previous assignments. ${ }^{10}$ The remaining columns show relative absorbances for $s-$ and p-polarized spectra $\left(A_{s}\right.$ and $A_{p}$ ), normalized to the $1500 \mathrm{~cm}^{-1}$ ODA breathing ring mode for both spin-cast and vapor deposited films.

Table I. Mode assignments and relative intensities for the s- and p-polarizations for the spin cast and VDP films. In the first column, "d" refers to the less ordered and "o" refers to the more ordered phases of the films. In the second column, $v$ refers to the stretching modes and $\delta$ refers to the bending (deformation) modes. In the second column, the orientation assignments are as follows ${ }^{10}$ :

A: parallel to the polymer chain axis

B: parallel to the repeating zigzag unit.

$C$ : normal to the zigzag unit and in plane of the rigid PMDA unit

D: normal to the zigzag unit and out of plane of the rigid PMDA unit

\begin{tabular}{|c|c|c|c|c|c|c|c|}
\hline \multirow{2}{*}{$\begin{array}{c}\text { Wavenumber } \\
\left(\mathrm{cm}^{-1}\right)\end{array}$} & \multirow[b]{2}{*}{ Mode/Orientation } & \multicolumn{3}{|c|}{ Spin Cast } & \multicolumn{3}{|c|}{ VDP } \\
\hline & & $A_{s}$ & $A_{p}$ & $A_{p} / A_{s}$ & $A_{S}$ & $A_{p}$ & $A_{p} / A_{s}$ \\
\hline $\begin{array}{l}1730(d) \\
1720(0)\end{array}$ & $v_{\text {CO }}$ asym $/ C$ & $\begin{array}{l}0.275 \\
0.840\end{array}$ & $\begin{array}{l}0.435 \\
1.110\end{array}$ & $\begin{array}{l}1.58 \\
1.32\end{array}$ & $\begin{array}{l}0.336 \\
0.888\end{array}$ & $\begin{array}{l}0.528 \\
0.937\end{array}$ & $\begin{array}{l}1.57 \\
1.05\end{array}$ \\
\hline 1500 & $v_{C C}(O D A) / B$ & 1.000 & 1.000 & 1.00 & 1.000 & 1.000 & 1.00 \\
\hline 1240 & $v_{C O C}$, asym $/ A$ & 0.648 & 0.599 & 0.93 & 0.614 & 0.589 & 0.96 \\
\hline $\begin{array}{l}1122(d) \\
1114(0)\end{array}$ & $\begin{array}{l}\delta, \mathrm{CH} \text { in-pln } / \mathrm{C} \\
\text { (ODA) }\end{array}$ & $\begin{array}{l}0.088 \\
0.175\end{array}$ & $\begin{array}{l}0.120 \\
0.196\end{array}$ & $\begin{array}{l}1.37 \\
1.12\end{array}$ & $\begin{array}{l}0.092 \\
0.180\end{array}$ & $\begin{array}{l}0.114 \\
0.180\end{array}$ & $\begin{array}{l}1.24 \\
1.00\end{array}$ \\
\hline $\begin{array}{l}827(d) \\
820(0)\end{array}$ & $\begin{array}{l}\delta, \mathrm{CH} \text { out of-pln } \mathrm{D} \\
\text { (ODA) }\end{array}$ & $\begin{array}{l}0.092 \\
0.138\end{array}$ & $\begin{array}{l}0.110 \\
0.164\end{array}$ & $\begin{array}{l}1.19 \\
1.19\end{array}$ & $\begin{array}{l}0.102 \\
0.129\end{array}$ & $\begin{array}{l}0.111 \\
0.127\end{array}$ & $\begin{array}{l}1.09 \\
0.98\end{array}$ \\
\hline 725 & $\delta$, co out of-pln $/ D$ & 0.168 & 0.198 & 1.18 & 0.169 & 0.174 & 1.03 \\
\hline
\end{tabular}

\section{DISCUSSION}

Anisotropy can be determined by comparing absorbances for in- and out-of plane electric fields as referenced to the $1500 \mathrm{~cm}^{-1}$ vibration that defines the direction of the zigzag unit (Fig. 4). In the analysis below, we assume no in-plane anisotropy, as evidenced by both refractive index and infrared transmission measurements. For instance, for the spin cast films, the D modes at 725, 820 and $827 \mathrm{~cm}^{-1}$ are stronger for the p-polarization (normal to the substrate) compared to the s-polarization. For VDP films this orientation is considerably less pronounced. These intensity ratios suggest that spin-cast films prefer to orient with the zigzag chains parallel to the substrate. For the VDP films, the data suggest that the zigzag chains have no preferred orientation. While the data could also suggest that the VDP films assume an orientation 
intermediate to "standing up" and "lying down", we have no reason to believe that such orientation would be preferred.

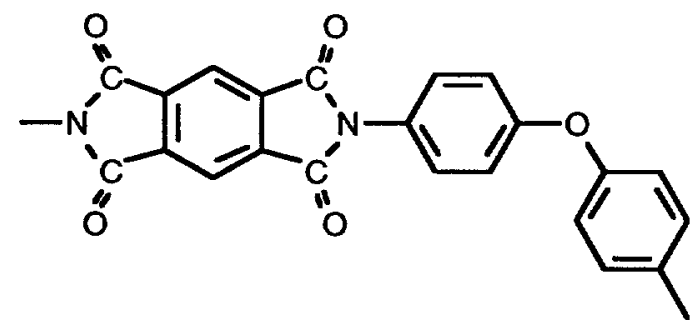

Fig. 4. A repeating zigzag unit of the PMDA-ODA polyimide polymer chain.

Analysis of the C-modes allows determination of the orientation of the plane of the phenyl ring and the pyromellitimide moiety with respect to the substrate. We will first consider the spin cast films whose zigzag chains have a preferential in-plane orientation. For the disordered regions, the $A_{p} / A_{S}$ ratio is $>1$, suggesting that in these regions, the plane of the phenyl rings and the pyromellitimide moieties is oriented at large angles with respect to the substrate plane. For the ordered regions, the $A_{p} / A_{s}$ ration is closer to 1 , suggesting that in the ordered regions, the plane of these groups is closer to the substrate plane.

Even though VDP films are more isotropic, the ATR FTIR spectra still reveal the presence of more and less crystalline regions within the film. VDP films may have shorter length molecular chains, thus resulting in smaller size crystalline regions. The presence of these crystalline regions can be observed from the examination of the asymmetric carbonyl stretch for the VDP films (Fig. 5). The higher frequency component (disordered region) is more pronounced in the p polarized spectrum (Fig. 5 $B)$. The data suggest that in VDP films, similarly to the spin cast films, the more ordered regions contain molecules with preferential alignment of the pyromellitimides along the substrate plane.

It is interesting to compare our results to a previous reflection absorption FTIR study of vapor deposited PMDA-ODA films by lida, et. al ${ }^{10}$. By referencing their spectra to a KBR pellet of commercial polyimide, the authors have observed a strong anisotropy for their vapor deposited films with the film orientation decreasing as the deposition rate increased. Since their highest deposition rates, $0.3 \mu \mathrm{m} / \mathrm{hr}$, are an order of magnitude lower than the rates employed in this study, their data are consistent with our results. These data seem to suggest that by increasing the deposition rates, the anisotropy can be significantly reduced.

\section{CONCLUSIONS}

We have compared the anisotropy of spin cast and vapor deposited PMDA-ODA polyimide films. In general, spin cast films were found to be more anisotropic than vapor deposited films. The polyimide zigzag chains within the spin cast films tend to align preferentially parallel to the substrate. In VDP films, no preferential orientation of the zigzag chains with the respect to the substrate could be resolved. In general, both VDP and spin cast films were found to contain crystalline and amorphous regions. Within the crystalline regions, the molecules were found to align with the plane of the phenyl ring and the pyromellitimide moiety tends parallel to the substrate. 

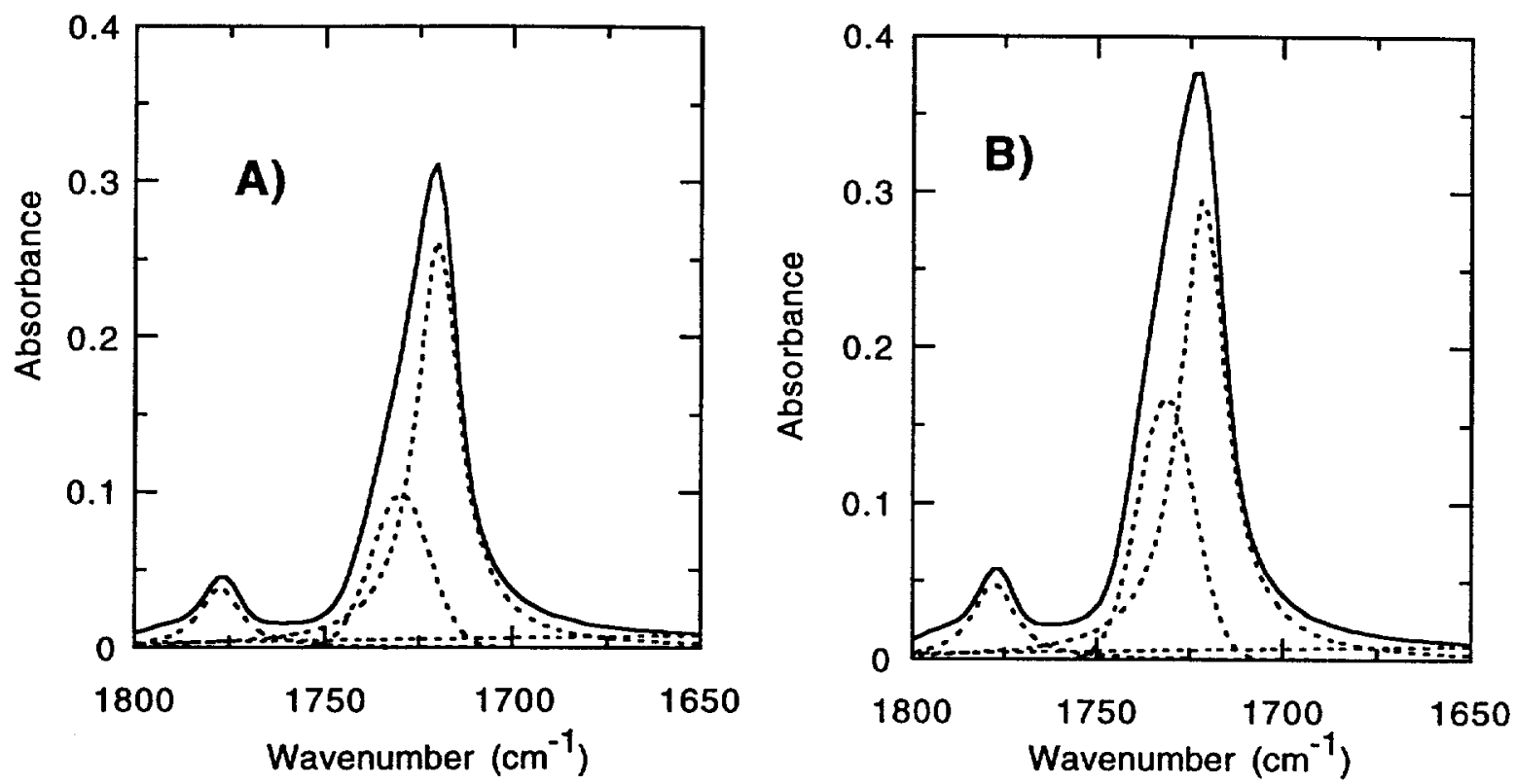

Fig. 5. ATR FTIR absorbance spectra for the carbonyl stretching region of the VDP films. A) s polarization, B) $p$ polarization.

\section{ACKNOWLEDGMENTS}

The authors would like to thank R. T. Graff for manufacturing the fixture used in ATR experiments and T. Neff of II-VI, Inc. for providing ZnSe samples for chemical compatibility studies. This work was performed under the auspices of the U.S. DOE by LLNL under contract no. W-7405-Eng-48.

\section{REFERENCES}

1. M. lijima, Y. Takahashi, U. S. Patent 4624867 (1986)

2. J. R. Salem, F. O. Sequeda, J. Duran, W. Y. Lee and R. M. Yang, J. Vac. Sci. Technol. 4, 369 (1986).

3. R. J. Jensen and J. H. Lai, in Polymers for Electronic Applications, edited by J. H. Lai, (CRC Press, Boca Raton, Florida, 1989) p. 33.

4. A. Deutsch, M. Swaminathan, M. H. Ree, C. W. Surovic, G. Arjavalingam, K. Prasad, D. C. McHerron, M. McAllister, G. V. Kopcsay, A. P. Giri, E. Perfecto and G. E. White, IEEE Transactions on Components, Packaging and Manufacturing Technology, Part B: Advanced Packaging 17, 486 (1994).

5. M. T. Pottiger, J. C. Coburn and J. R. Edman, J. Polym. Sci. B: Polym. Phys. 32, 825 (1994).

6. N. Takahashi, D. Y. Yoon and W. Parrish, Macromolecules 17, 2583 (1984).

7. V. Liberman, V. Malba and A. F. Bernhardt, to be published(1996).

8. H. Ishida, S. T. Wellinghoff, E. Baer and J. L. Koenig, Macromolecules 13, 826 (1980).

9. D. Boese, H. Lee, D. Y. Yoon, J. D. Swalen and J. F. Rabolt, J. Polym. Sci. B: Polym. Phys. 30, 1321 (1992).

10. K. lida, Y. H. Chen, S. Nakamura, and G. Sawa, Proc. IEEE Conf. on Electr. Ins. and Diel. Phenom., 731 (1994). 


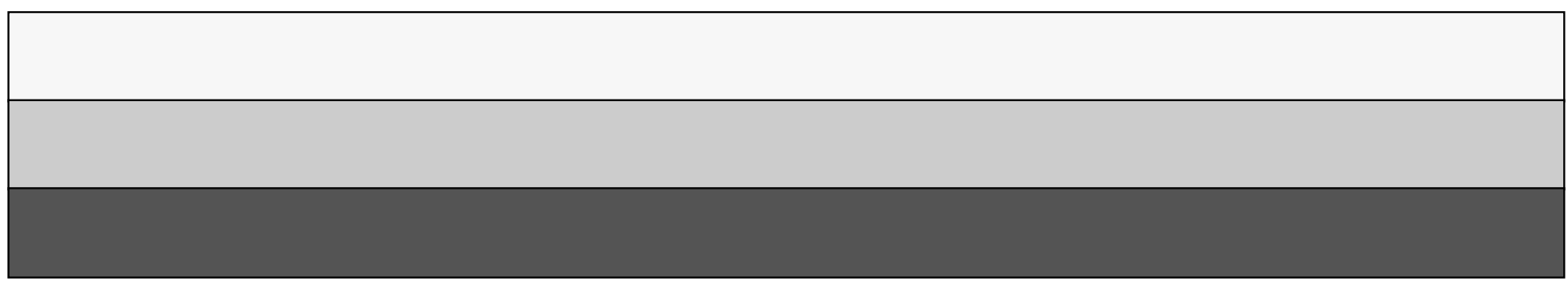

